\title{
Low-LER Tin Carboxylate Photoresists using EUV
}

\author{
Ryan Del Re, ${ }^{\text {a }}$ Miriam Sortland, ${ }^{\mathrm{a}}$ James Passarelli, ${ }^{\mathrm{a}}$ Brian Cardineau, ${ }^{\mathrm{a}}$ Yasin Ekinci, \\ Michaela Vockenhuber, ${ }^{\mathrm{c}}$ Mark Neisser, ${ }^{\mathrm{d}}$ Daniel A. Freedman, \\ and Robert L. Brainard ${ }^{\mathrm{a} *}$ \\ ${ }^{a}$ College of Nanoscale Science and Engineering, Albany, NY 12203 \\ ${ }^{\mathrm{b}}$ State University of New York at New Paltz, New Paltz, NY 12561 \\ ${ }^{\mathrm{c}}$ Paul Scherrer Institut, Villigen, Switzerland \\ ${ }^{\mathrm{d}}$ Sematech, Albany NY 12203
}

\begin{abstract}
Pure thin films of organotin compounds have been lithographically evaluated using extreme ultraviolet lithography (EUVL, $13.5 \mathrm{~nm}$ ). Twenty-one compounds of the type $\mathrm{R}_{2} \mathrm{Sn}\left(\mathrm{O}_{2} \mathrm{CR}^{\prime}\right)_{2}$ were spin-coated from solutions in toluene, exposed to EUV light, and developed in organic solvents. Exposures produced negativetone contrast curves and dense-line patterns using interference lithography. Contrast-curve studies indicated that the $\mathrm{E}_{\max }$ values were linearly related to molecular weight when plotted separately depending upon the hydrocarbon group bound directly to tin ( $\mathrm{R}=$ butyl, phenyl and benzyl). Additionally, $\mathrm{E}_{\max }$ was found to be linearly related to free radical stability of the hydrocarbon group bound directly to tin. Dense-line patterning capabilities varied, but two resists in particular show exceptionally good line edge roughness (LER). A resist composed of an amorphous film of $\left(\mathrm{C}_{6} \mathrm{H}_{5} \mathrm{CH}_{2}\right)_{2} \mathrm{Sn}\left(\mathrm{O}_{2} \mathrm{CC}\left(\mathrm{CH}_{3}\right)_{3}\right)_{2}(\mathbf{1 3})$ achieved $1.4 \mathrm{~nm}$ LER at $22 \mathrm{~nm}$ halfpitch patterning and a resist composed of $\left(\mathrm{C}_{6} \mathrm{H}_{5} \mathrm{CH}_{2}\right)_{2} \mathrm{Sn}\left(\mathrm{O}_{2} \mathrm{CC}_{6} \mathrm{H}_{5}\right)_{2}$ (14) achieved $1.1 \mathrm{~nm}$ LER at $35 \mathrm{~nm}$ halfpitch at high exposure doses $\left(600 \mathrm{~mJ} / \mathrm{cm}^{2}\right)$. Two photoresists that use olefin-based carboxylates, $\left(\mathrm{C}_{6} \mathrm{H}_{5} \mathrm{CH}_{2}\right)_{2} \mathrm{Sn}\left(\mathrm{O}_{2} \mathrm{CCH}=\mathrm{CH}_{2}\right)_{2}$ (11) and $\left(\mathrm{C}_{6} \mathrm{H}_{5} \mathrm{CH}_{2}\right)_{2} \mathrm{Sn}\left(\mathrm{O}_{2} \mathrm{CC}\left(\mathrm{CH}_{3}\right)=\mathrm{CH}_{2}\right)_{2}$ (12), demonstrated much improved photospeeds $\left(5 \mathrm{~mJ} / \mathrm{cm}^{2}\right.$ and $\left.27 \mathrm{~mJ} / \mathrm{cm}^{2}\right)$ but with worse LER.
\end{abstract}

\section{Keywords:}

EUV, Organometallic, Resist, Photoresist, Tin, LER, Line Edge Roughness, LWR, Line Width Roughness

\section{Introduction}

Traditional chemically-amplified photoresists have been used in the microelectronics industry for over twenty years. ${ }^{1,2}$ These lithographic systems have enormous capability and have achieved the photolithographic performance that allowed the industry to follow Moore's law. ${ }^{3}$ However, the cost and complexity of achieving smaller feature sizes is becoming more difficult. Extreme ultraviolet lithography (EUVL, $13.5 \mathrm{~nm}$ ) continues to rely on conventional chemically amplified photoresists, but many challenges

Extreme Ultraviolet (EUV) Lithography VI, edited by Obert R. Wood II, Eric M. Panning,

Proc. of SPIE Vol. 9422, $942221 \cdot$ (C) 2015 SPIE · CCC code: 0277-786X/15/\$18

doi: $10.1117 / 12.2086597$

Proc. of SPIE Vol. $9422942221-1$ 
exist that must be resolved before its successful implementation. One major challenge in successful EUV implementation is photoresist design. ${ }^{4,5}$

EUV photons $(92 \mathrm{eV})$ interact with matter differently than do $193-\mathrm{nm}$ photons $(6.4 \mathrm{eV})$, and therefore, the fundamental design of photoresists may need to change to obtain the best lithographic performance for EUVL. As we decrease film thickness to compensate for smaller patterning without line collapse, ${ }^{6}$ organic films may be unable to absorb the photons efficiently ${ }^{7}$ which may lead to poorer sensitivity and poorer line edge roughness. ${ }^{8}$ Etch resistance may also be inadequate in these thinner, organic films. ${ }^{9,10}$ We have previously reported on a new resist system, MORE (Molecular Organometallic Resists for EUV), that seeks to overcome these potential obstacles. By using elements that have high EUV optical density, we intend to create molecular films that greater numbers of EUV photons than purely organic resists and to create resists with better resolution, sensitivity and LER.

Here, we present recent lithographic results of several MORE mononuclear tin compounds that have been spin coated as thin films on wafers and exposed to EUV light. We have synthesized a series of tin compounds of the general type $\mathrm{R}_{2} \mathrm{Sn}\left(\mathrm{O}_{2} \mathrm{CR}\right)_{2}$ in an attempt to study the effects of increasing ligand bulk and increasing Sn-R bond energy on photosensitivity and lithographic performance. Although the sensitivity of many of these compounds is poor $\left(E_{\text {size }}=50-600 \mathrm{~mJ} / \mathrm{cm}^{2}\right)$, they are capable of good resolution and excellent LER. In particular, compound $\mathbf{1 3}$ (Figure 1A) resolves 16-nm half-pitch dense lines with $2.1 \mathrm{~nm}$ LER and resolved 22-nm half-pitch dense lines with an LER of $1.4 \mathrm{~nm}$ (Figure 1B). We also see a linear trend when we compare molecular weights with $\mathrm{E}_{\max }$ within a particular compound family (Figure $2 \mathrm{~B}$ ).

(A)

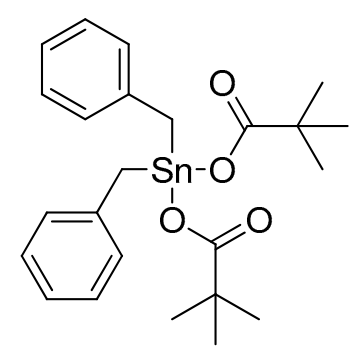

13
(B)
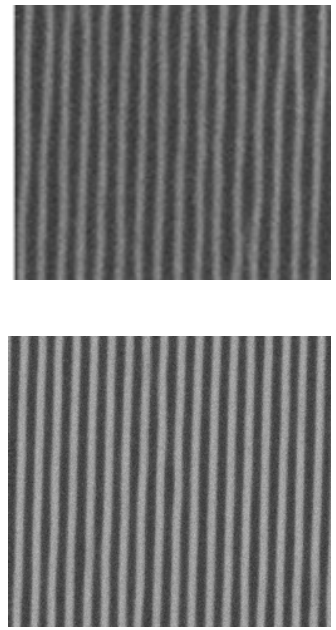

Resolution: $16 \mathrm{~nm}$

LER: $\quad 2.1 \mathrm{~nm}$

Dose: $\quad 600 \mathrm{~mJ} / \mathrm{cm}^{2}$

Resolution: $22 \mathrm{~nm}$

LER: $\quad 1.4 \mathrm{~nm}$

Dose: $\quad 600 \mathrm{~mJ} / \mathrm{cm}^{2}$

Figure 1. (A) Structure of Compound 13, $\left(\mathrm{C}_{6} \mathrm{H}_{5} \mathrm{CH}_{2}\right)_{2} \mathrm{Sn}\left(\mathrm{O}_{2} \mathrm{C}\left(\mathrm{C}\left(\mathrm{CH}_{3}\right)_{3}\right)\right)_{2}$. (B) Dense line patterning capabilities of compound 13 exhibiting low LER. 


\section{Results and Discussion}

A series of compounds of the type $\mathrm{R}_{2} \mathrm{Sn}\left(\mathrm{O}_{2} \mathrm{CR}\right.$ ') have been prepared through modified literature procedures. $^{11,12}$ Toluene solutions of these compounds were spin-coated onto silicon wafers as thin films. The resists were then exposed to EUV light at the Paul Scherrer Institut (PSI) for evaluation of both contrast curves and dense line and space patterning. The exposed wafers were developed in hexanes for 5-25 seconds revealing negative-tone imaging. Photosensitivity was quantified by finding the $\mathrm{E}_{\max }$ (energy needed to achieve maximum thickness after development) from the contrast curves and patterning was evaluated using $\mathrm{E}_{\text {size }}\left(\mathrm{mJ} / \mathrm{cm}^{2}\right)$, line edge roughness $(\mathrm{LER}, \mathrm{nm})$, and resolution $(\mathrm{nm})$.

\subsection{Photosensitivity $\left(E_{\max }\right)$ Dependence on Molecular Weight.}

Contrast curve exposures of resists at PSI gives a good measure of their relative photosensitivities. Previously, Cardineau, et al., ${ }^{13}$ reported that the sensitivities of tin-oxo clusters improve as the molecular weight of the counter ions decrease. We tested a series of mononuclear $\mathrm{R}_{2} \mathrm{Sn}$ (carboxylate) $)_{2}$ compounds and found little correlation when plotting $\mathrm{E}_{\max } v s$. molecular weight of the compounds (Figure 2A). However, we did see a molecular weight dependence with good correlation when we plotted $\mathrm{E}_{\max } v s$. carboxylate variations with $\mathrm{R}$ held constant (Figure $2 \mathrm{~B}$ ). We think that this relationship between $\mathrm{E}_{\max }$ and $\mathrm{MW}$ merely indicates that efficiency of the negative-tone behavior (e.g. cross-linking) will increase as the intermolecular distances between tin centers decreases.

\section{2 $\mathbf{E}_{\text {size }}$ Dependence on Molecular Weight.}

We patterned dense lines with interference lithography at PSI and found that the same linear dependence held between molecular weight and $\mathrm{E}_{\text {size }}$ with dibenzyl tin dicarboxylates that are non-acrylate based. Interestingly, dibenzyl tin diacrylate (11) and dibenzyl tin dimethacrylate (12) deviated from this linear trend (Figure 3) and are much more sensitive than would be predicted based on molecular weight arguments alone. In this case, the deviation may be attributed to a different and more efficient mechanism of exposure.
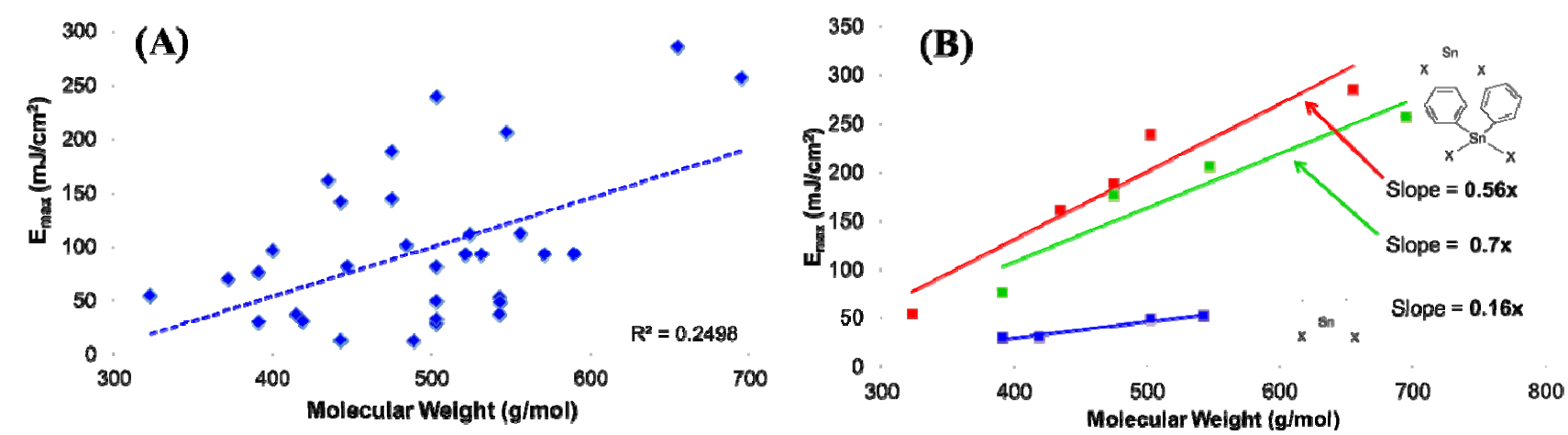

Figure 2. (A) Comparing a wide variety of organotin carboxylates shows little correlation of data, however, (B) strong correlations exist when comparing a particular family of compounds. 


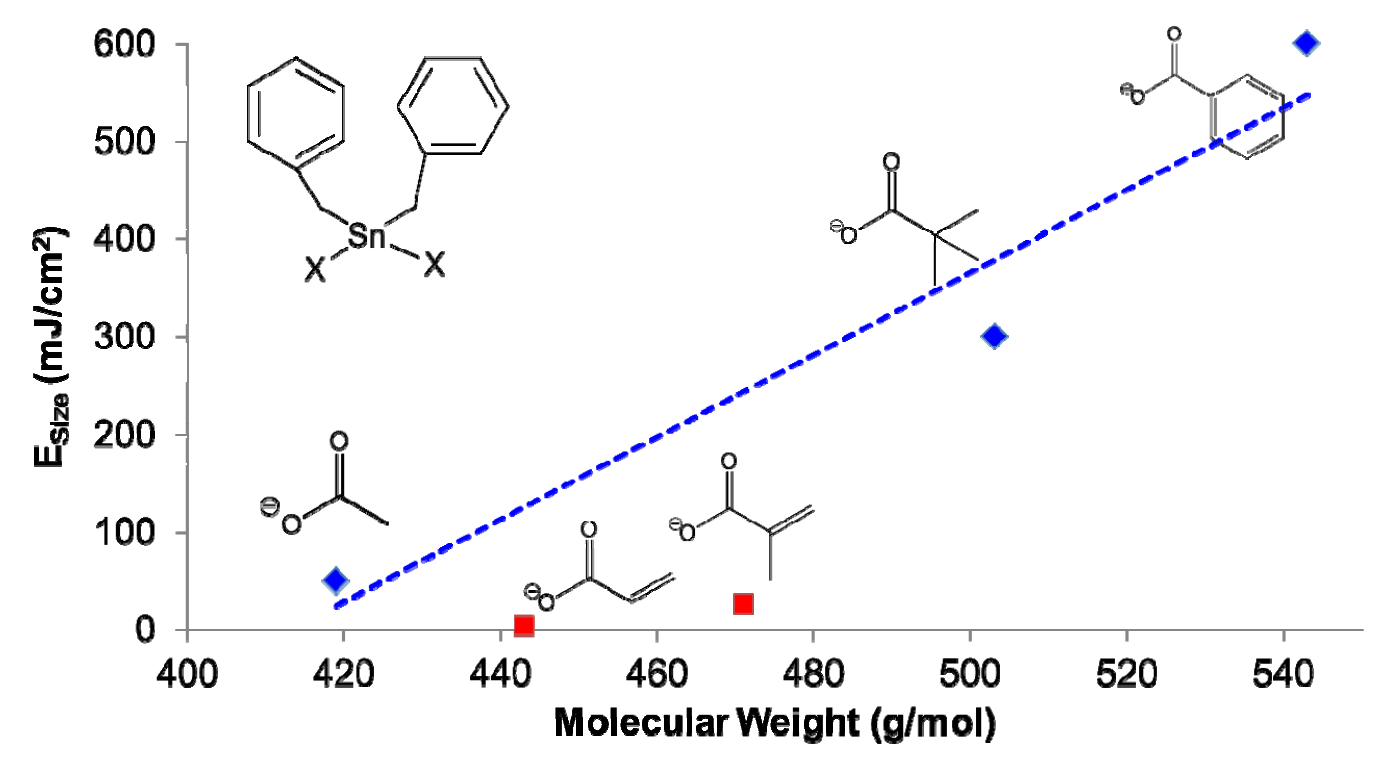

Figure 3. Patterning shows the same linear dependence with a significant deviation when analyzing acrylate based resists.

\subsection{Tin- Carbon Bond Energy and $\mathrm{E}_{\max }$ Dependence.}

Figure 4 shows the effects of modified tin-carbon bond energy. Here, we analyze $\mathrm{E}_{\max }$ of compounds $\mathbf{4}$, 13, and 19: $\mathrm{R}_{2} \mathrm{Sn}\left(\mathrm{O}_{2} \mathrm{C}\left(\mathrm{C}\left(\mathrm{CH}_{3}\right)_{3}\right)_{2}\right.$ where $\mathrm{R}=$ phenyl, butyl, benzyl. There are no experimental measurements available of direct bond energies between $\mathrm{Sn}-\mathrm{R}$ in these cases, but Cottrell ${ }^{14}$ has measured the $\mathrm{C}-\mathrm{H}$ bond energies and we used these values as a measure of relative free radical stability. The inverse relation suggests that photolytic cleavage occurs at the Sn-C bond during exposure. Decreasing this bond energy may help improve photospeeds.

\subsection{Imaging of Tin Dicarboxylates.}

Imaging was performed at the Swiss Light Source (SLS) at PSI using interference lithography. ${ }^{15}$ Figure 5 shows dense line/space patterns of three dibutyl-tin compounds. Although these resists exhibit pattern modulation to $16 \mathrm{~nm}$, the film quality is poor. Figure 6 shows $(\mathrm{Bu})_{2} \mathrm{Sn}\left(\mathrm{O}_{2} \mathrm{CCH}_{3}\right)_{2}(2)$ at increasing levels of magnification. At low magnification, many light-colored spots are present in the exposed areas. At higher magnification, dense-line patterns are visible. All of the dibutyl-tin complexes required high exposures and had poor LER. 


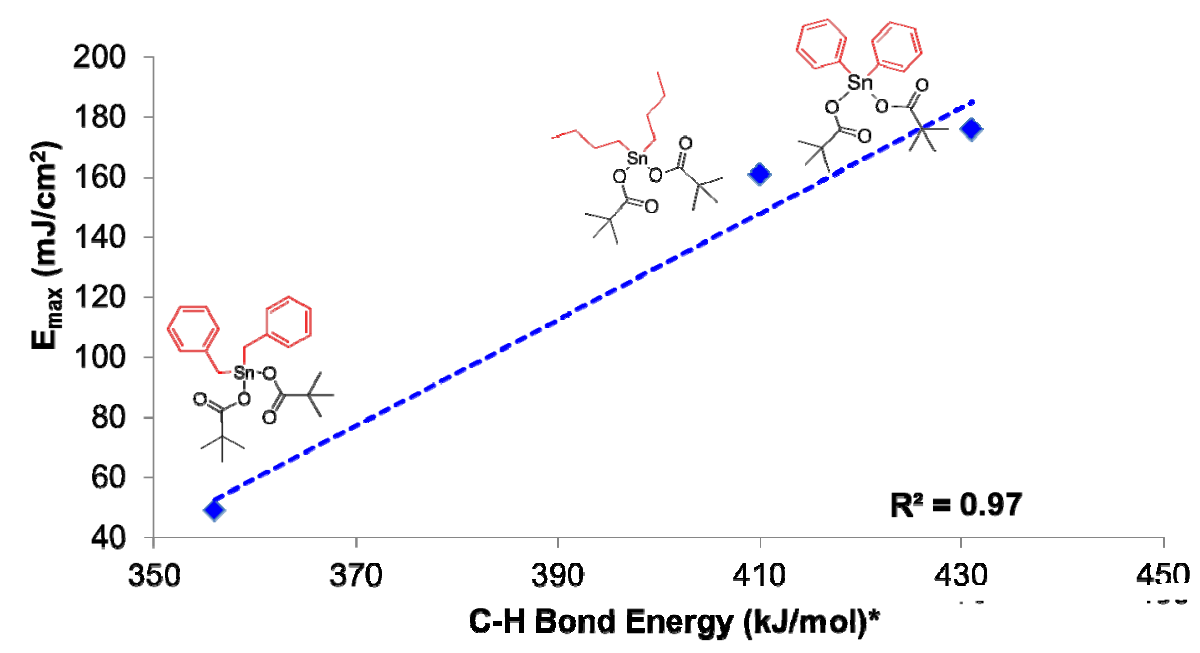

Figure 4. Variation of tin-carbon bond energy shows an inverse relationship with sensitivity. This may indicate that this bond energy plays an important role during photoexposure.

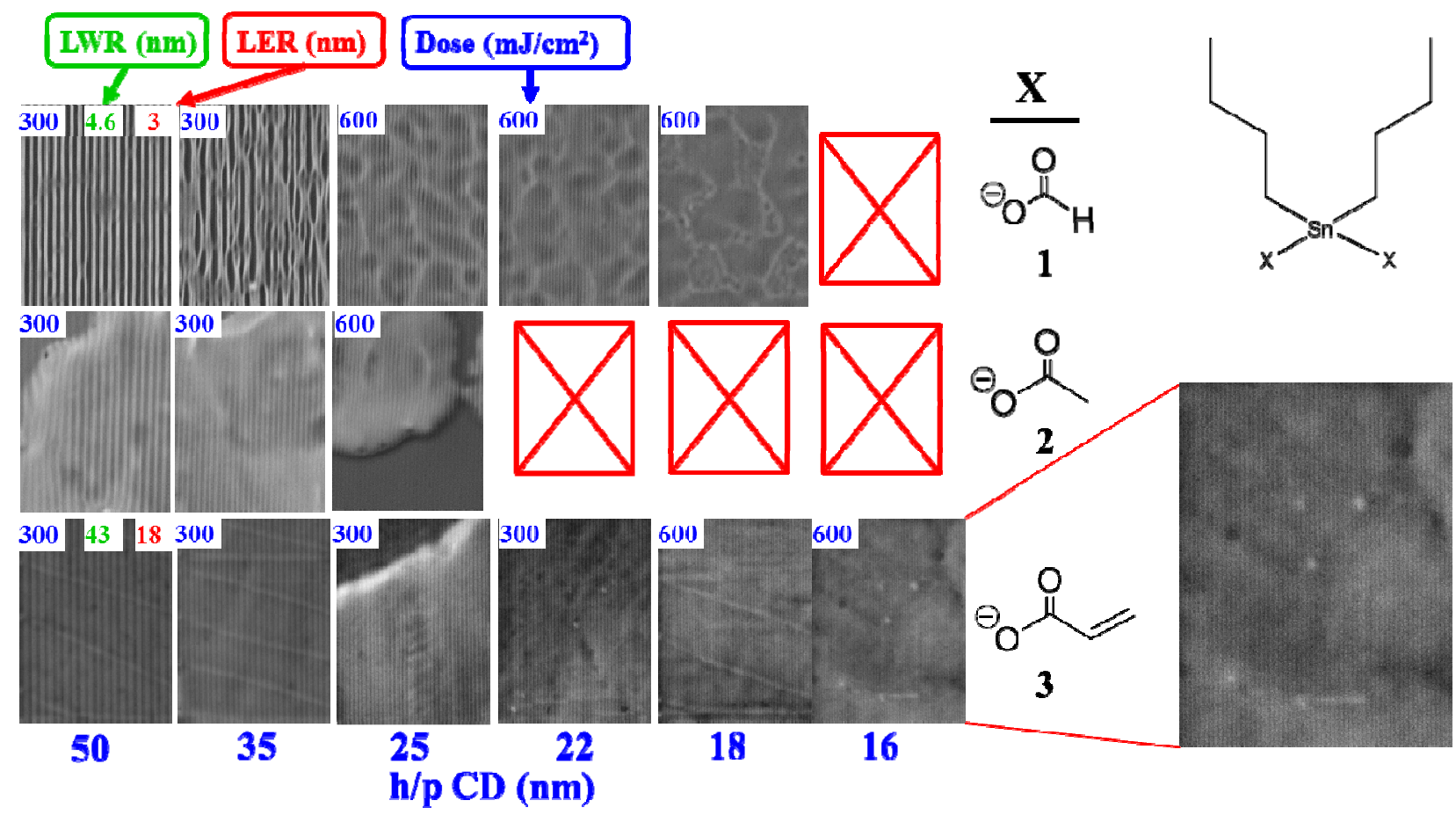

Figure 5. Dense lines patterning capabilities of dibutyl tin dicarboxylates. All of the films exhibited poor film quality after development. 

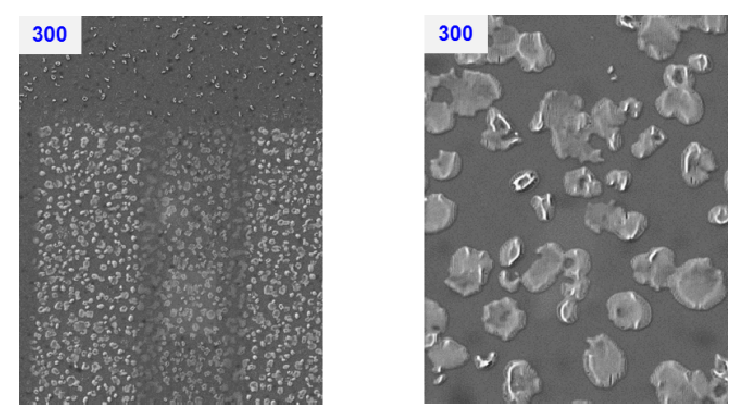

$3 k x$

$20 \mathrm{kx}$

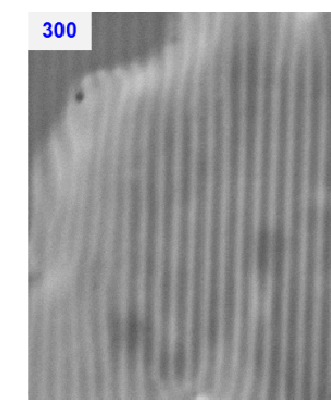

$150 \mathrm{kx}$

Figure 6. Strange patterning phenomenon of compound $\left(\mathrm{C}_{4} \mathrm{H}_{9}\right)_{2} \mathrm{Sn}\left(\mathrm{O}_{2} \mathrm{CCH}_{3}\right)_{2}$ (2). Each island shows patterning with no patterning in the spaces.

Figure 7 shows dense line/space patterning capabilities of various dibenzyl tin compounds. Photoresponse has a large range of values $\left(50-600 \mathrm{~mJ} / \mathrm{cm}^{2}\right)$ corresponding to a wide range of resolutions. One compound (13) has achieved $16 \mathrm{~nm}$ dense patterning at a high exposure dose of $600 \mathrm{~mJ} / \mathrm{cm}^{2}$. The most remarkable quality of two of these dibenzyl tin resists is the extremely low LER, $1.4 \mathrm{~nm}$ (Compound 13) and $1.1 \mathrm{~nm}$ (Compound 14) (Figure 8).

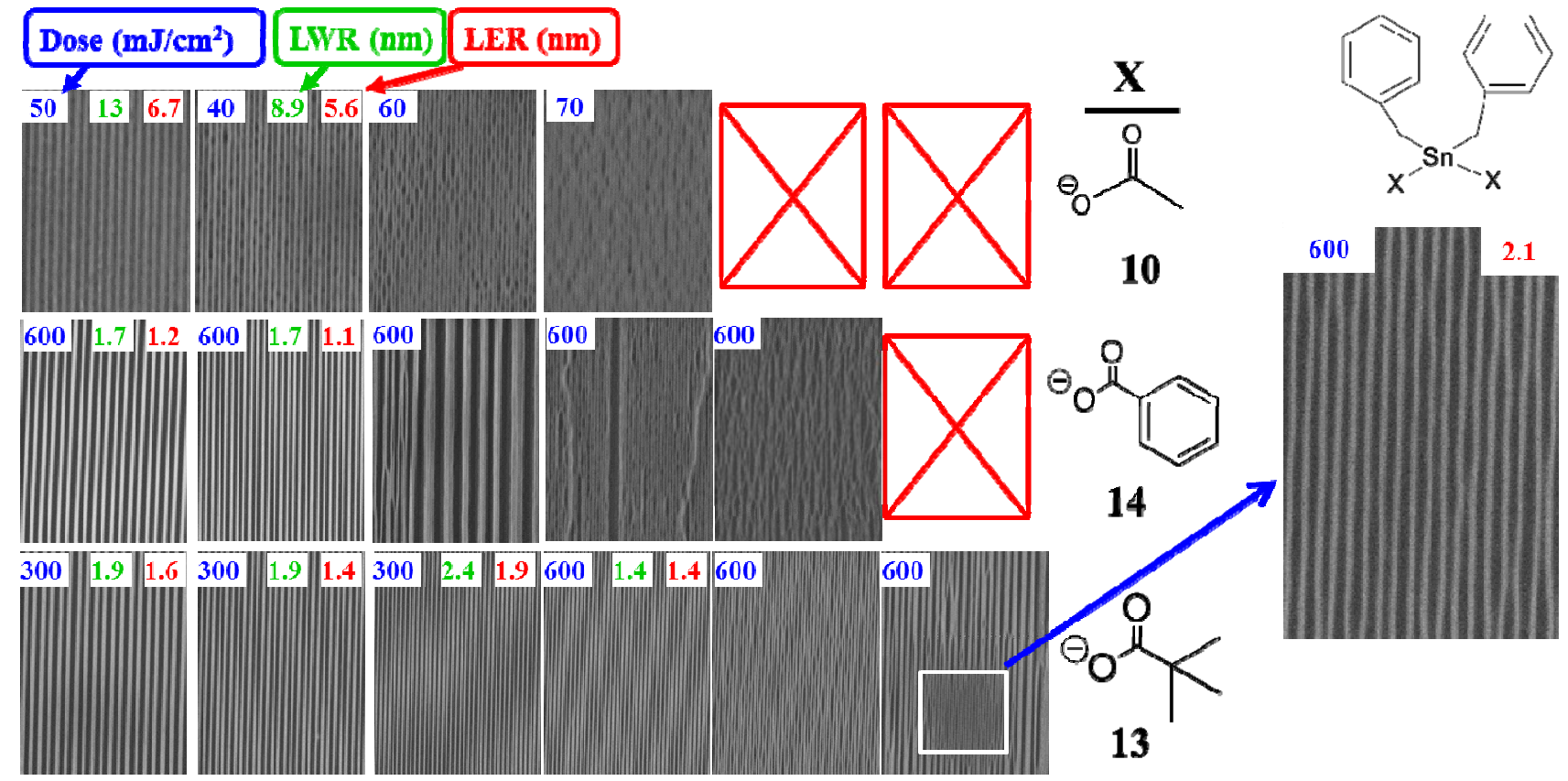

Figure 7. Dense lines patterning capabilities dibenzyl tin dicarboxylates. These resists achieved the highest resolution and lowest LER of any metal based resist evaluated by our group. 


\subsection{Low LER Resists}

We have patterned dense lines of some of these compounds and have resolved features to $16 \mathrm{~nm}$ with significant pattern collapse at high exposure doses $\left(600 \mathrm{~mJ} / \mathrm{cm}^{2}\right)$. Perhaps the most remarkable quality observed is the extremely low LER of two resists $(1.4 \mathrm{~nm}$ and $1.1 \mathrm{~nm}$, Figure 8). Although the exposure doses in most cases were extremely high, we discovered two molecules $(\mathbf{1 1}, \mathbf{1 2})$ that required much lower exposure doses $\left(5 \mathrm{~mJ} / \mathrm{cm}^{2}, 27 \mathrm{~mJ} / \mathrm{cm}^{2}\right)$ to pattern dense lines (Figure 9). We propose that these molecules undergo a more efficient exposure mechanism, possibly utilizing a free radical polymerization after an initiation event.
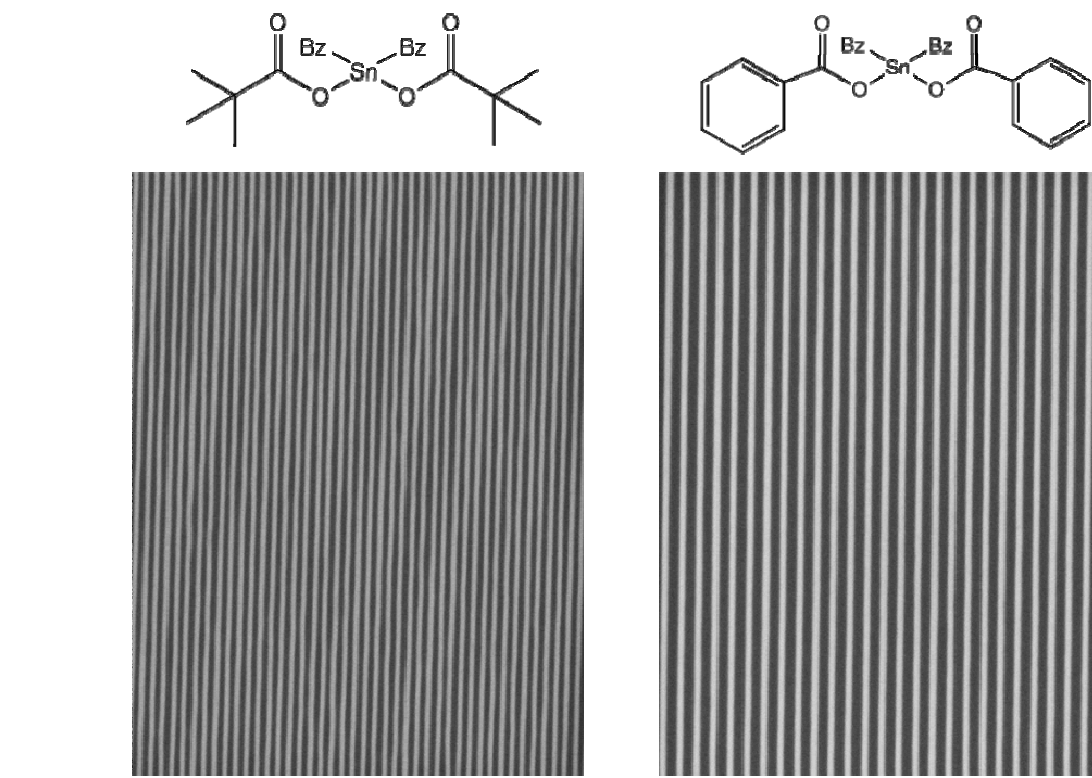

Compound:

CD:

LER:

LWR:
13

$22 \mathrm{~nm}$

$1.4 \mathrm{~nm}$

$1.4 \mathrm{~nm}$

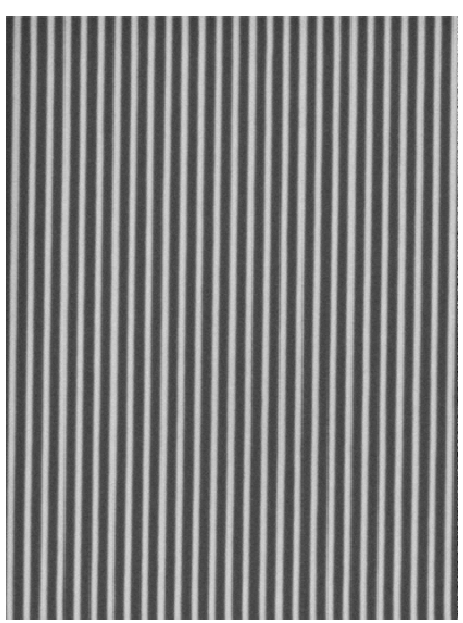

14

$35 \mathrm{~nm}$

$1.1 \mathrm{~nm}$

$1.7 \mathrm{~nm}$

Figure 8. Compounds $\mathbf{1 3}$ and $\mathbf{1 4}$ display high resolution patterning with extremely low LER. 


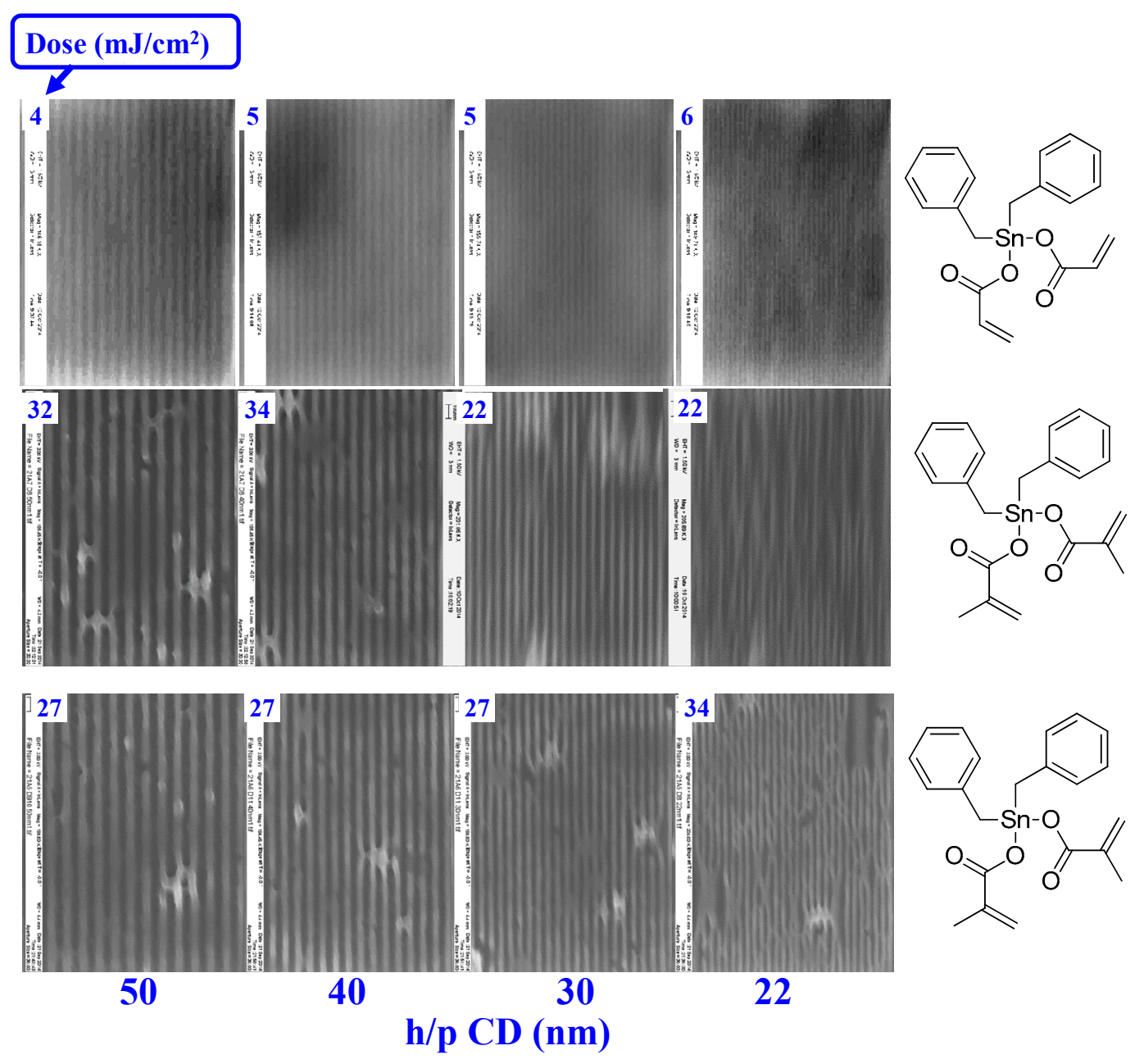

Figure 9. Dibenzyl tin acrylates displayed much higher photospeeds at a cost of resolution and LER.

\section{Conclusions}

We have evaluated the EUV lithographic capabilities of twenty-one organotin dicarboxylates. We systematically evaluated the imaging capabilities of these compounds as a function of the identity of the alkyl and carboxylic acid groups. We discovered a general trend within three classes of compounds $(\mathrm{R}=$ phenyl, butyl, benzyl) that showed improving sensitivities with decreasing molecular weight. Variations of the organic substituent bound directly to tin (phenyl, butyl, benzyl) showed a correlation between resist sensitivity and the stability of the corresponding organic free radical. We concluded that the alkyl-tin bond strength is very important in determining the ultimate sensitivity and is the most likely bond that is cleaved 
during exposure. Increased molecular weight due to carboxylate variations may affect the resist sensitivity by either reducing the films mass density and EUV optical density or by acting as physical spacers that reduce intermolecular interactions after exposure.

We have patterned dense lines of some of these compounds and have resolved features to $16 \mathrm{~nm}$ with significant pattern collapse at high exposure doses $\left(600 \mathrm{~mJ} / \mathrm{cm}^{2}\right)$. Perhaps the most remarkable quality observed is the extremely low LER of two resists $(1.4 \mathrm{~nm}$ and $1.1 \mathrm{~nm}$, Figure 8$)$. Although the exposure doses in most cases were extremely high, we discovered two molecules $(\mathbf{1 1}, \mathbf{1 2})$ that required much lower exposure doses $\left(5 \mathrm{~mJ} / \mathrm{cm}^{2}, 27 \mathrm{~mJ} / \mathrm{cm}^{2}\right)$ to pattern dense lines (Figure 9). We propose that these molecules undergo a more efficient exposure mechanism, possibly utilizing a free radical polymerization after an initiation event.

\section{Acknowledgments}

We would like to thank Sematech for financial support of this work. We would also like to thank Dupont for supplying us with ESCAP polymer and King Industries for supplying us with a thermal acid generator for organic underlayers. Finally, we would like to thank Patrick Naulleau at the Lawrence Berkeley National Laboratory for providing us with SuMMIT Lithography Image Analysis software.

\section{Experimental Methods}

General. All reactions were carried out under a nitrogen environment. The reagents n-dibutyltin dichloride, n-dibutyltin hydroxide oxide, and diphenyltin dichloride were purchased from Alfa Aesar. All other reagents were purchased from Sigma Aldrich. All reagents were used as received unless specified. Dibenzyltin dibromide (8) was prepared by a literature procedure. ${ }^{16}$

Resist Formulation and Imaging. Resist formulations were made by dissolving solids in toluene and filtering through a $0.2 \mu \mathrm{m}$ PTFE filter. Formulations were spin-coated a custom underlayer (crosslinked hydroxyethyl methacrylate/methyl methacrylate copolymers) on 4-inch silicon wafers. 40-nm to 60-nm thick resist films were made by adjusting spin-speed and formulation concentration. Resist films were then exposed with 13.5-nm radiation and developed with a hexanes developing solution. Exposures were performed at Paul Scherrer Institut on the XIL-II beamline using interference lithography.

SEM Metrology. Scanning electron microscope micrographs were collected at Paul Scherrer Institut on a Zeiss Supra VP55. Accelerating voltage was set between 1-2 KeV and the working distance was $4 \mathrm{~mm}$. All images were at either $150 \mathrm{kx}$ or $200 \mathrm{kx}$ magnification.

LER Analysis. All LER values were obtained from the analysis of the SEM micrographs in SuMMIT Lithography Image Analysis software. 


\section{References}

1. Ito, H., IBM J. Res. Develop (2000) 44 (1/2), 119.

2. http://www.chemheritage.org/discover/media/magazine/articles/25-3-patterning-the-world.aspx?page=1.

3. Moore, G., Electronics (1965) 38.

4. http://www.itrs.net/Links/2013ITRS/2013Chapters/2013Litho.pdf.

5. Sakai, K., et al., Progress of high index immersion exposure system. In 5th International Symposium on Immersion Lithography Extensions, The Hague, Netherlands, (2008)

6. Brainard, R. L., et al., Journal of Photopolymer Science and Technology (2001) 14 (4), 531.

7. Gullikson, E., Center for X-Ray Optics (CXRO) Filter Transmission. http:/henke.lbl.gov/optical_constants/filter2.html, (1995-2010)

8. Neissar, M., et al., Journal of Photopolymer Science and Technology (2012) 25, 87.

9. Paul, J., et al., The Electrochemical Society Abstract (2012).

10. Paul, J., et al., Advanced Etch Technology for Nanopatterning II, Proceedings from SPIE (2013) 8685, 86850V.

11. Sandhu, G., et al., Journal of Organometallic Chemistry (1987) 321, 15.

12. Meriem, A., et al., Applied Organometallic Chemistry (1991) 5, 195.

13. Cardineau, B., et al., Microelectronic Engineering (2014) 127, 44.

14. Cottrell, T., The Strengths of Chemical Bonds, 2d ed., Butterworth, London, 1958.

15. http://www.psi.ch/lmn/euv-interference-lithography.

16. Boudjouk, P., et al., Chemistry of Materials (1996) 8, 1189. 